\title{
Real Models are really on M0 - or how to make Programmers use Modeling
}

\author{
Joachim Fischer ${ }^{1}{ }^{a}$, Birger Møller-Pedersen ${ }^{2} \mathbb{C}^{\mathrm{b}}$ and Andreas Prinz ${ }^{3} \mathbb{C}^{\mathrm{c}}$ \\ ${ }^{1}$ Department of Computer Science, Humboldt University, Berlin, Germany \\ ${ }^{2}$ Department of Informatics, University of Oslo, Oslo, Norway \\ ${ }^{3}$ Department of ICT, University of Agder, Grimstad, Norway \\ fischer@informatik.hu-berlin.de,birger@ifi.uio.no,andreas.prinz@uia.no
}

\begin{abstract}
Keywords: model, system, language, ontological modeling
Abstract: $\quad$ This paper discusses the term 'model' and the role of the level $\mathrm{M} 0$ in the four-layer metamodeling architecture of MOF/OMG. It illustrates the failures of the OMG MOF standard and how a model is an abstraction, not a description. We apply two simple approaches: (1) observing the use of models (of real or planned systems) in system development, including prototyping, simulations, and models in general, and (2) comparing modeling with programming. These approaches lead to the conclusion that models should be placed on M0, while UML models are model descriptions. This conclusion leads to a better understanding of InstanceSpecification for description of snapshots, and of metamodeling applied to ontologies.
\end{abstract}

\section{INTRODUCTION}

Modeling and programming communities are diverging, each developing their own languages and tools ${ }^{1}$.

Programmers mainly focus on (program) executions, while modellers handle different kinds of what in the modeling community are called models (domain models, requirements and design models) in a mixture of diagrams and text. Programmers are to a certain extent also modeling, but in order to have programmers apply modeling for real, (and to have modellers also focus on executions), there has to be a shared understanding of what models are.

The notions of models and metamodels from the (UML) modeling community do not help. As long as the four-levels OMG/MOF architecture [OMG, 2016] with the levels M0 to M3 has existed, the role of M0 has been discussed, questioned, misunderstood, or even ignored [Atkinson and Kühne, 2003, Bézivin and Gerbé, 2001, Eriksson et al., 2013, Favre, 2004].

In this paper, we will contribute to a shared under-

\footnotetext{
a (iD https://orcid.org/0000-0003-2476-3996

b (iD https://orcid.org/0000-0003-2123-3260

c (D) https://orcid.org/0000-0002-0646-2877

${ }^{1}$ Domain-specific languages (DSL) [Fowler, 2005] and Problem Solving Environments (PSE) [Kawata, 2015] are approaches that bring modeling and programming together in specific domains.
}

standing by looking at the use of models in systems development, thereby giving the right role to models.

The ultimate goal of systems development is to produce systems, i.e. changing sets of executing objects that interact with each other and with entities in the environment of the systems. Systems are made using various kinds of descriptions, ultimately programs from which executions with objects (on level M0) are created. Similarly, execution of Executable $U M L$ [OMG, 2018] models will generate a structure of objects at M0. That is, Executable UML models are also descriptions (prescriptions) of executions.

We will also look at the general notion of model. It is generally agreed that a model is an abstraction of an existing or planned system. A model is made from a perspective and has a purpose. Therefore, only a subset of the properties of a system are represented in the model. It is possible to experiment with the models instead of with the real systems and deduce properties - for good reasons, it is not possible to experiment with planned systems. This idea of model is independent of the model being material or mathematical or digital. In programming (and in modeling with Executable UML), experiments are not experiments with the prescriptions, but experiments are done with the executions (the systems).

The arguments above indicate that models are at $M O$, while descriptions and prescriptions are on lev- 
els M1-M3. We are aware that this is different from the common terminology, where descriptions and prescriptions on M1 are called (UML) models. However, placing models on M0 matches how programmers and system developers think about models, and it matches the everyday use of the term model.

In [Madsen and Møller-Pedersen, 2018], the authors have made the same argument, i.e. that program executions are models. We will apply this understanding to the MOF architecture, which is not addressed in [Madsen and Møller-Pedersen, 2018].

The (UML) modeling community has different opinions on M0: [Kühne, 2006] has the reality to be modeled there, while UML in 2011 [OMG, 2011] had InstanceSpecifications there. The understanding of having models at M0 provides a better understanding of UML InstanceSpecification, metamodeling, and domain modeling, including ontologies.

The paper is structured as follows. In Section 2, we discuss the role of models in systems development, including the general understanding of the concept 'model'. Section 3 compares modeling and programming. Section 4 covers different understandings of InstanceSpecification, including its basis for ontological metamodeling, followed by a review of existing understandings of M0 in Section 5. Finally, we summarize in Section 6.

\section{SYSTEM DEVELOPMENT}

\section{System and System Description}

Systems development produces systems that fulfil a given purpose and provide required functionalities. For this paper, we assume that system development uses object-oriented programming and modeling.

Definition 1 (System). A system is a changing set of executing objects that interact with each other and with entities in the environment of the system. Objects may be existing entities like devices, and they may be entities that have to be made as part of the systems development. This way, a system is a set of possible executions, i.e. a set of object configurations that exist at different time points.

Systems are made using various kinds of system descriptions from which these executing objects are created, see the left side of Figure 1.

Among these kinds of descriptions, programs are prescriptions of systems as possible program executions. A program execution will consist of a changing structure of objects according to the prescriptions of

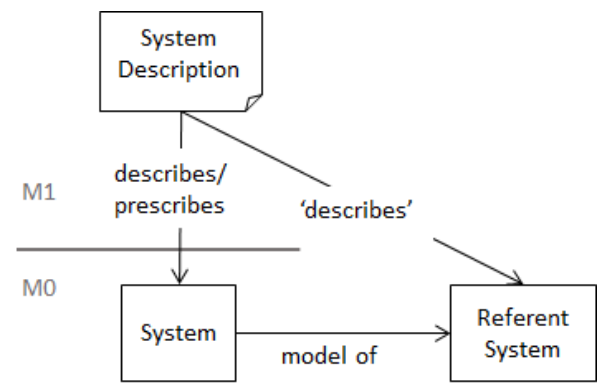

Figure 1: Description and Prescription, adapted from [Fischer et al., 2016]

objects and classes in the program, and these objects will obviously be on M0 and thereby the systems are at M0.

For Executable UML [OMG, 2018] the situation is the same: Executable UML model execution will generate a structure of objects on M0. That is, Executable UML models are really prescriptions of executions.

Definition 2 (Model). A model is a system that is in the model-of relationship to a referent system, existing or planned.

Note that both physical and mathematical models are systems, because it is their behaviour (their executions) that makes them models. Scale models are also concrete representations, but typically with the same object structure that does not change over time.

As can be seen in Figure 1, a system description always leads to a system, which is a set of possible executions. The system does not need to be a model if there is no related referent system.

Figure 1 is based upon the system and model concepts from the language DELTA ${ }^{2}$ ( [Holbæk-Hanssen et al., 1973]) placed in the context of the MOF fourlayer architecture. Note that the system (the execution of the system description) is at M0, whereas the referent system (the system to be modeled) is not part of this architecture. Still, it is placed at the level of M0 to indicate that this is an existing or planned real system, not a description.

A UML model is not a model according to this definition of model, but rather a model description. We will use the term 'UML model' to denote model descriptions expressed in UML.

Experiments with systems, like testing and simulations, are experiments with an execution, not with descriptions. Validation is the process of finding out whether a system has the right model-of relation to an existing or planned real system, i.e. whether their executions match. Even for review, it may be interesting

\footnotetext{
${ }^{2}$ DELTA was a very early (1973) modeling language, at that time called 'system description language'.
} 
to compare, e.g. required sequence diagrams with the sequence diagrams of the system (for a given functionality), as reported in [Daun et al., 2019], where the generated sequence diagrams are called 'review models'.

Another kind of experiment is prototyping. In [Exner et al., 2014], it is well documented that prototyping is experimenting with systems in varying degrees of completeness, not experimenting with descriptions. Validation by prototyping is the process of finding the system that is the desired model of a planned system.

\section{Modeling in Systems Development}

In system development with modeling languages, a system description will typically be a graphical description of a planned system combining new elements with existing (real) elements. The example in Figure 2 of a temperature controlling system exemplifies this. It describes a class of systems consisting of existing (white) elements like Heating and Cooling actuators, and a Temperature sensor; the (grey) Controller is the one to be made so that it fits into this architecture.

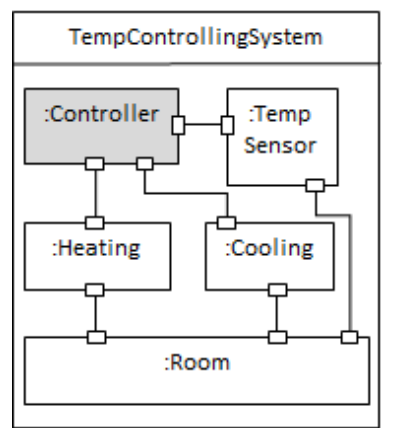

Figure 2: UML diagram that describes a temperature controlling system (from [Fischer et al., 2016])

Note that Figure 2 is not a picture of a class of systems, but a UML description of a composite class with parts and connectors.

Sensors and actuators model the real sensors and actuators. A simulation will simulate the Room, the sensors and the actuators, while the Controller will be tested or experimented with. In this process, the description of the Controller will probably be changed according to the simulations. However, it is still the running Controller that is the model of the controller to be in a building when it is deployed.

\section{Programming in Systems Development}

If only programming languages are exploited in system development, the situation is the same. Some class descriptions are descriptions of concepts from the application domain (like real passengers, flights, seats, tickets), and objects of these classes model the corresponding passengers, flights, seats etc. Other class descriptions are descriptions of elements of the planned system (e.g. elements of the planned reservation system). Few programming languages support the kind of description in Figure 2. However, there is no fundamental reason for not being able to express this in programming languages, see, e.g. [Aldrich et al., 2002].

When it comes to systems as introduced in Figure 1 , programmers do not doubt that they are part of system development (which of course includes more than programming) where the goal is to produce systems by making programs that are executed by computers.

For Executable UML the experiments will also be with executions, not with diagrams, so real Executable UML systems really reside on level M0. There is no difference between program executions and executions for Executable UML, except for the different abstraction levels of the language mechanisms. For Executable UML the artefacts at M1 are therefore descriptions of systems.

What then about the parts of UML that are not covered by Executable UML, e.g. interactions and use cases, and that are still used in system development? They both reside at M1. Use cases are typically used as prescriptions of required functionality of the system, while interactions may be used as both prescriptions of required behaviour (or undesired behaviour) and descriptions of what happens with a system. So they are not models, according to the definition of model above. As mentioned above, in general, a UML model on M1 is a system description, or more precisely a description of a system that is a model of a referent system.

\section{Models in general}

The following definition of model in general summarizes definitions from Webster, Collins, Wikipedia, [Bossel, 2013], and a general understanding of model.

A model is a small or large, abstract or actual, representation of a planned or existing entity or system from a particular viewpoint and with a specific purpose.

Observe that this definition of model always defines a relationship between the system acting as the 
model, and the system being modeled, see also Figure 1 and Definition 2.

Mathematical models are examples of abstract representations. A mathematical model is usually a set of equations describing properties of a given system, e.g. in the form of differential equations.

Libraries are systems with changing sets of books and loans. Models of libraries with the purpose to understand libraries or to make computer-based library systems must be systems of objects representing real books and loans. The model, in this case, is an actual representation. Similarly, the model of the temperature controlling system in Figure 2 is an actual representation: it contains sensors and actuators that model the real sensors and actuators that are part of the real building when the Controller is deployed.

In [Madsen and Møller-Pedersen, 2018], the following illustrative example of a model as an actual representation is used. Consider the Mini in Figure 3. What is the model of the Mini: the Lego car to the

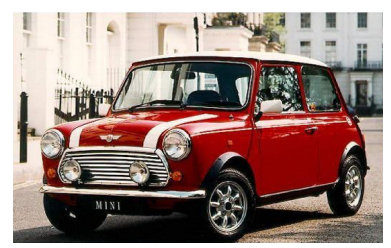

Figure 3: Mini

right in Figure 4 or the description of how to build it to the left in Figure 4? Any child will answer 'the Lego car'. Even grown-up people (they have been children too) will answer that the Lego car is the model of the real car.

Which of these is a model of the Mini?

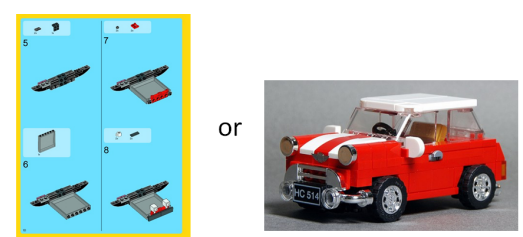

Figure 4: Description and Model

This example also illustrates the notion of interacting or experimenting with a model. A kid playing with the Lego car to imitate driving interacts with the model (the Lego car), its different states, like position, orientation, and velocity, while imitating how a car turns around. In case the Lego car has no engine and no steering control, playing with it simulates the execution. In case of a machine and steering capabilities, the kid will control the execution at crucial points, like changing orientation and speed. In case of a more elaborate Lego car (or even a Lego robot) that is prescribed to follow a given route and avoid obstacles, kids will experiment with the running model car by, e.g. placing or moving obstacles.

Some people get the chance to be trained as pilots in flight simulators. Flight simulators are models of real flights, and the pilots interact (maybe also experimenting) with the program execution (and some specialised hardware), not with the program that implements the simulator. Had it been done entirely in Executable UML, then the pilots would interact with the execution on level M0, not with the descriptions at M1. Descriptions of classes, state machines and activities as part of Executable UML are model descriptions, while the executions are models for interaction and experimentation.

\section{MODELING AND PROGRAMMING}

\section{Metamodels and Grammars}

It is established that Figure 5 gives the correspondence between the definition of modeling and programming languages, see, e.g. [Butting et al., 2018] and [Bézivin and Gerbé, 2001].

\begin{tabular}{|c|c|c|}
\hline M3 & meta-metamodel & EBNF \\
\hline M2 & metamodel & grammar \\
\hline M1 & (UML) model & program \\
\hline M0 & model execution & program execution \\
\hline
\end{tabular}

Figure 5: Metamodels and Grammars

This correspondence is obvious, provided that the modeling language is executable, like, e.g. Executable UML. However, even for UML in general this is the case: objects of classes defined in UML at M1 reside at $\mathrm{M} 0$.

However, metamodels and grammars are not completely the same. Metamodels describe abstract syntax (structure) of a language only and their concrete syntax usually is defined using editors. Grammars define the concrete syntax, and parsers generate abstract syntax trees from concrete syntax. Metamodels may include links, e.g. from the use of a concept to the definition of the concept. Parsers use attributes in the objects of the abstract syntax trees instead of such links. Even with the apparent correspondence between metamodels and grammars, the switch between them requires some effort, see again [Butting et al., 2018]. 


\section{Programs and UML Models}

A program (on M1) is a prescription of a number of possible program executions. A program execution will consist of a changing structure of objects according to the prescriptions of objects and classes in the program, and these objects will be on M0. Objects behave according to their prescription of behaviour as part of the program at M1.

As mentioned above, some objects, like passenger objects in a reservation system, will model the corresponding real passengers. Properties (like name and address) and capabilities (like changing address) of the passengers will be reflected by corresponding properties of the objects that model the passengers.

A similar approach should be taken for executable modeling languages, and for modeling languages in general. As an example: Executable UML model execution will generate a structure of objects according to the prescriptions of Executable UML classes at M1, and these objects will be on M0. Objects behave according to their prescription of behaviour as part of the Executable UML model at M1, i.e. Executable UML models are prescriptions of executions.

This approach divides the world into descriptions/prescriptions and objects, as shown in Figure 6.

\begin{tabular}{cr} 
M3 & Descriptions and prescriptions, in terms of \\
M2 & diagrams, text, or combined \\
M1 & Objects, with behaviours, states and \\
\hline M0 & links between objects
\end{tabular}

Figure 6: Descriptions and objects

Below the line, there are objects, there are states of objects, and there are state changes resulting from the behaviour of the objects. Objects model phenomena in the application domain.

Above the line, there are no objects, there are no states, and there are no state changes. However, there are prescriptions of objects (singular or by means of classes), there are prescriptions of states (e.g. descriptions of attributes of objects), and there are prescriptions of state changes, e.g. assignments and behaviour prescriptions in general.

In the following, we will use the term description for both description and prescription, except in cases where it is essential to distinguish. The main difference is between objects (at M0) and descriptions of objects (at M1-M3).

Compared with M0, not only M1 but also M2 and M3 contain descriptions. The fact that M1 and M2 have objects of metaclasses at the level above does not change this. These objects are there just for representing descriptions, just like a structure of linked objects represents programs in the form of abstract syntax trees. These objects are handled by tools (e.g. editors) that manipulate descriptions; they are not objects being parts of executions.

\section{Programmers and Modeling?}

How does the idea of model presented here relate to programmers, who more often than not live by the slogan 'Real programmers do not model'? They argue that making separate descriptions of what they can do in their favourite programming language is a waste of time. Besides, such descriptions often become obsolete because of inadequate tools with no two-way transformations.

However, programmers do model, but not in modeling languages. Programming involves modeling because application domain concepts are identified and represented by classes with properties that are given by the purpose of the system being developed. Objects of these classes model the corresponding phenomena. If the domain is library systems, there will be books, in programs described by a class Book and with Book objects being models of the real books, see Figure 7 for the situation with UML and Java. Starting with a UML class book, the Java class is generated from a class description in UML. However, this does not make the UML description a model of the Java code; a model has to be a system, not just a description. Similarly, in a reservation system, there will be

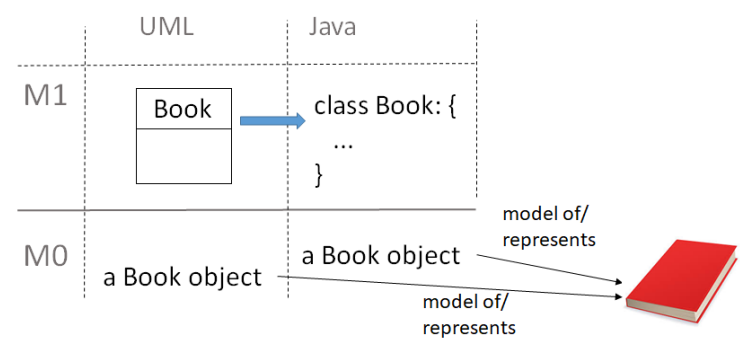

Figure 7: UML and Java class at M1, objects at M0 modeling the real book

Passenger and Flight objects that model the corresponding real passengers and flights.

It is important to emphasize that the UML class Book is not a model of the Java class Book. Instead, both of them are prescriptions of objects that are models of the real books.

This way, a shared understanding of models and modeling can (in addition to better tool support) help programmers use modeling. A programmer would not call the program (Figure 5) a model, as the program is 'just' a prescription. The ultimate goal is 
the program execution. As illustrated above, the program executions (library system, reservation system) will have objects that model the corresponding elements from the application domain and even from reality. So, for programmers, the models are on M0. Of course, a program execution contains elements that are there just to have the execution on a specific platform or because of the implementation technique used. Some of these elements are simply platform- or implementation specific. However, there may also be elements that are modeled according to some technical domain, like, e.g. communication using a specific protocol.

\section{Semantics and Meaning}

There is a strong connection between level $\mathrm{M} 0$ and the semantics of a modeling or a programming language. Moreover, there is a relation that relates the model with the real referent system it models, which we have called model-of.

Let us look into semantics. Any formal language provides a semantics detailing the transition from a (system) description into a system. In the metamodeling architecture, this is the move from descriptions on levels M1-M3 to objects and executions on level M0. Therefore, (formal) semantics is a relation, and it is vertical (crossing levels). The level crossing might not happen in the first step, as, e.g. with translational or denotational semantics. However, someplace down the line, the description has to come to life, crossing the level. We generalize the idea of semantics as follows.

Definition 3 (Semantics). Semantics is the relation between a (system) description and its prescribed possible executions (the system) ${ }^{3}$. Sometimes, the describe relationship can be considered semantics.

It is important to note that the language defines the semantics - in fact, this is the only aspect of the language crossing the level boundaries. So the language description on level M2 details the transition of a description on level M1 into a system on level M0. The user is then free to create descriptions on M1 in the language, which will be turned into executions (systems) on M0 by way of the semantics. This way, semantics is defining linguistic instantiation as defined in [Kühne, 2006]. Moreover, it also defines the semantics of ontological instantiation in case the language provides it (see Section 4 for more details).

\footnotetext{
${ }^{3}$ The DELTA language report used the neutral term 'generator' that generates a system based upon a system description. A generator could be a machine or a human being, or a mixture.
}

The model-of relation is different from semantics. There can be many models of the same system. Even though the real systems are outside the metamodeling architecture, they are at the same level as the executions in the architecture, which reside at M0. So it is most meaningful to consider the real referent systems on level M0 as well. This way, the model-of relation is horizontal, informal and connected to abstraction.

\section{Behaviour Descriptions for Metaclasses}

With the above understanding of models as systems on level M0, and with just descriptions at M1, what is then the understanding of metamodels at M2? M1 contains descriptions of objects at M0. The descriptions are represented by objects (of classes at M2), so M2 similarly contains descriptions. In relation to M1, they are descriptions, while in relation to M0, they are meta-descriptions.

Metamodeling allows defining abstract syntax for languages, and the levels M1 to M3 express this well. As we shall see below, modeling ontologies is not metamodeling [Aßmann et al., 2006]. Languages for modeling ontologies, like OWL [W3C, 2012], may, however, be defined by metamodels. OMG has, in fact, defined the language ODM [OMG, 2014] for defining ontologies.

Definition 4 (Metamodel). A metamodel is a description of a language. The corresponding executions belong to an implementation of the language, i.e. an IDE. The relation between a metamodel on M2 and its execution on $M 1$ is the same as the relation between descriptions on M1 and models on MO, namely semantics.

The narrow definition of metamodel in OMG would only allow structural definitions of languages. However, there could also be behaviour descriptions as part of metaclasses, e.g. metaclass Class. Such behaviour would not be M0-behaviour common to all instances of all classes of the metaclass Class. Two classes A and B, both represented by objects of the metaclass Class, would not get the behaviour of metaclass Class. Instead, as objects of metaclasses are instances that represent descriptions, then operations on these objects would typically change the objects, and thereby the description represented by these objects. This could, e.g. be operations to be used in refactoring of descriptions.

Such behaviour is executed on the level M1, i.e. with tools handling descriptions. The most obvious tools here would be editors and IDEs, with functionality for changing descriptions. In this case, the descriptions on level M1 would be changing, such that the 
description that is executed here would be the metamodel on M2, which is a model of the language execution (the editor or the IDE). The same thought is used in language workbenches [Fowler, 2005].

\section{UNDERSTANDING INSTANCESPECIFICATIONS}

\section{InstanceSpecifications as Snapshots}

We have established a distinction between the world of descriptions and the world of systems of objects, see figure 6. For programming and for Executable UML, the objects will be elements of executions (systems), and as such, they do not belong to the description stack of M1-M3. These systems at M0 are created using prescriptions. Most often, they should model a referent system. In case an execution does not match the corresponding execution in the referent system, the usual way is to use some debugging tool to provide descriptions (snapshots) of objects as they are at a particular stage of execution.

Definition 5 (Snapshot). A snapshot is a description of the current state of execution. This way, a snapshot relates to an execution semantics and has to be able to represent all instances existing at runtime 4 .

UML provides Instancespecifications for expressing snapshots, see Figure 8. An InstanceSpecification will have a slot for each of the features of the object, describing the current value of the feature. Instancespecifications are de-

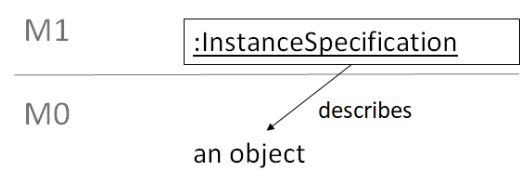

Figure 8: Instance Specification

scriptions at M1 for describing objects at M0. An Instancespecification may, however, also be used to prescribe objects, see the following quote from [OMG, 2011].

An Instancespecification may specify the actual existence of an instance in a modeled system. Alternatively, an InstanceSpecification may provide an illustration or example of a possible instance in a modeled system.

\footnotetext{
${ }^{4} \mathrm{~A}$ debugger is a tool that can show the current state of execution in some notation, which we call snapshot.
}

Is then InstanceSpecification enough to form the basis for making snapshots? InstanceSpecifications can represent instances of classes. However, at runtime, there are also instances of other entities, for example, methods, variables, and threads.

The name InstanceSpecification was deliberately chosen over the name Object of the early versions of UML. One reason was that Object was often confused with objects at M0. Another reason was that it is now possible to use it for describing other kinds of instances. An Instancespecification has a classifier that is the classifier of the instance being described, as illustrated in Figure 9. The classifier

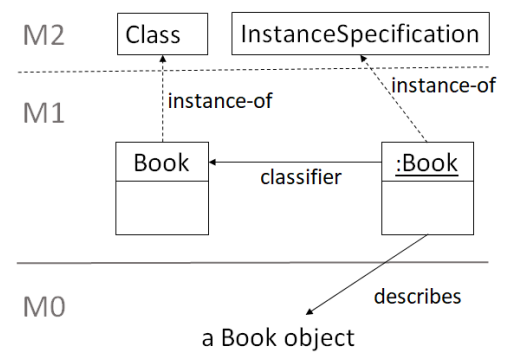

Figure 9: Instance Specification with classifier

property of InstanceSpecification may refer to Behaviours (as Behaviour is a subclass of Class which in turn is a subclass of Classifier). Method is a Behaviour associated with an operation of a class, so an InstanceSpecification may also be a description of a method invocation instance, commonly called activation record.

An activation record of a method call includes values for its attributes. However, it also includes the current stage of execution, a so-called dynamic link to the activation record of the calling method (forming a call stack). A similar case is the so-called static link of an object of a class going to the object of the enclosing scope.

In principle, an InstanceSpecification could represent the current state of execution, the static and dynamic links and other required elements as slots. However, the slots are related to the features of the classifier, and it is evident that classifiers do not have features for the particular elements described above. The classifier property of InstanceSpecification only denotes the classifier of the instance.

Moreover, a snapshot must be able to indicate how far the execution has come. In implementations of programming languages this is represented by a program counter, a link into the behaviour description, to the action (e.g. as part of a method description) currently being executed. UML is somewhat more general in that a classifier behaviour may be an activity or a state machine. In the last case, the correspondence 
to program counter would, e.g. be the current state, but the current stage of execution may also be a point in a transition.

These elements belong to the instantiation semantics of the classifier, see [Prinz et al., 2016]. UML uses its built-in instantiation semantics for classes, but this does not extend to the general case of classifiers being something else than classes. This built-in semantics - missing the extra elements is also valid for InstanceSpecification, which makes it not in itself sufficient for general snapshots. It would, however, be straight forward to use Instancespecification to convey the values of features according to its classifier, and then provide dynamic link and static link associated with the InstanceSpecifications.

\section{Ontological Instances}

The classifier relation from an InstanceSpecification to, e.g. a Class (see Figure 9) is by some authors called ontological instance-of, see [Atkinson and Kühne, 2003, Kühne, 2006]. As a consequence, the notion of ontological metamodeling is introduced, leading to a different understanding of M0 than ours. As an example, in [Atkinson and Kühne, 2003] the Lassie InstanceSpecification is said to model the real Lassie, see Figure 10. The InstanceSpecification Lassie is correctly

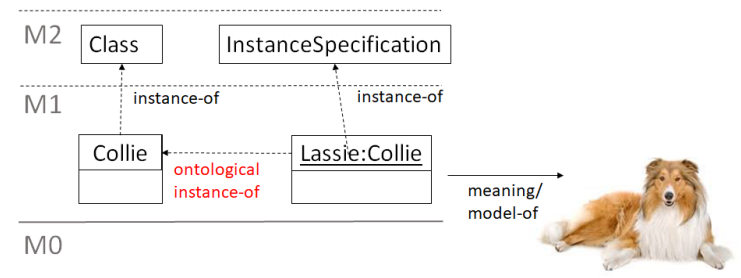

Figure 10: Ontological instance-of as defined in [Atkinson and Kühne, 2003]

placed on level M1 and is an instance of the M2 metaclass Instancespecification, which was the metaclass Object at the time of [Atkinson and Kühne, 2003]. In addition, Lassie is said to be an (ontological) instance of the class Collie, thus being an instance of two classifiers. Objects of the class Collie as part of model execution at M0 have no role in ontological metamodeling.

We have seen that for programming (and also for Executable UML), the objects that model real objects from the domain (like real passengers, real flights) are objects at M0 and not at M1, and there is no reason to distinguish between Executable UML and plain UML on this issue. With this understanding, the ob- ject that really models the real Lassie is a Collie object at M0. The Lassie is just a description of the Collie object that exists as part of the execution at M0, see Figure 11. The relation between

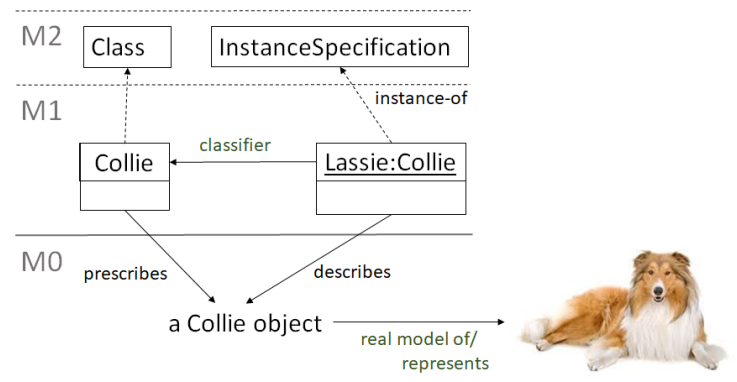

Figure 11: The real model of Lassie

the Lassie instance specification and Collie is that of classifier. Note that the classifier attribute of Lassie does not make it an instance of the class Collie. Instances of class Collie reside in executions at M0; Lassie is an instance at M1 of the metaclass InstanceSpecification at M2.

It is claimed that there is a paradox between linguistic and ontological instantiation in that ontological classes have to be linguistic instance-of a metaclass on level M3, thereby having a linguistic instance-of crossing two levels, as ontological classes are on level M1.

The example used to illustrate this is the notion of breed. In a linguistic view, Collie is a class at M1, that is an instance of the metaclass Class on level M2. Applying an ontological view, the class Collie is said to be an ontological instance-of a class Breed, making Breed a kind of metaclass residing at a higher level than Collie. This way, ontological modeling becomes metamodeling even though it takes place at the M1 level.

We will use a programming viewpoint to resolve (in fact, dissolve) the paradox because things become more evident when applied to a real case. It is striking that the whole breed discussion does not relate to a real application. In a real application, the number of objects will change during the execution. There will be so many objects ${ }^{5}$, that it would not be meaningful to make an InstanceSpecification for each of them in order to describe the execution.

Programs reside on M1, even though programmers do not think in these terms. Part of programming is modeling in the sense that domain concepts are identified and represented by classes, and specialized concepts by means of subclasses. This kind of

\footnotetext{
${ }^{5}$ Considering all possible executions, there are infinitely many objects to consider.
} 
modeling from programming is not different from the kind of modeling that is applied when using modeling languages.

For a programmer, Breed will be a class describing the characteristics of breeds, and there may be subclasses for the various breeds. Dog will be an abstract subclass of class Canine, and it will have a Breed property as follows.

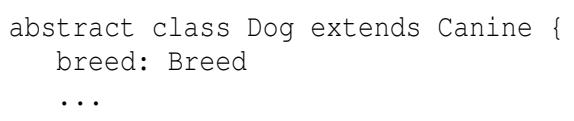

Even though there are no pure Dog objects, all dogs have a breed, therefore the property breed of the class Dog. Breed is the type of the property breed, and the various values are defined, e.g. as elements of an enumeration or as subclasses of Breed. A Collie is thereby a subclass of Dog where breed=CollieBreed for all objects of class Collie (i.e. it cannot be changed for individual Collie objects), and each collie is an instance of the class Collie. There may be other properties of Dog, and these may be set for the different instances of Collie. In languages with class attributes, i.e. attributes that apply to all instances of a class, breed would be such an attribute. The value breed=CollieBreed assigned to breed will be assigned upon creation of a objects and from then on being constant/final. Note that we assume the language to only support single inheritance, so Dog cannot be said to be classified as both a Canine and a Breed. With multiple inheritance, it would be possible to classify in both directions.

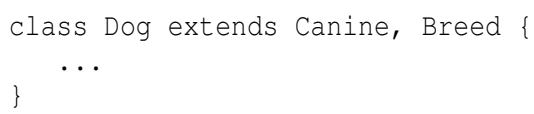

Still, this would not help, as the superclass Breed would have to be a kind of 'virtual superclass' in order to specify that for Collie the superclass Breed really should be CollieBreed (a subclass of Breed) and not Breed. Very few languages support virtual superclasses, so programmers that are modeling will apply inheritance for the primary classification and then use properties for secondary characterization.

One may argue that it is not obvious what should be the primary classification and what should be properties. However, modeling always uses a particular viewpoint and a specific purpose, and these will determine the primary classification. In [Eriksson et al., 2013] a 'language use approach' is applied to solve the above mentioned paradox. They also conclude that there is no paradox, and they use the terms 'substantial types' for the primary classification and 'moment types' for the types of properties. Ontologies also make a distinction between classes of objects and properties of these objects, so there would be no problem in defining an ontology where breed is a property.

Another argument against Breed being a class and class Collie being an instance of this is that dogs as we know them not only have different breeds, they also have different roles:

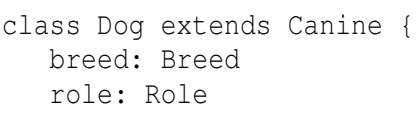

There are, e.g. pet dogs, guide dogs, watchdogs, shepherd dogs, sledge dogs. Different breeds are suited for different roles. For example, the collie breed is suitable for shepherding.

To be complete, the breed attribute is really typed by a Breed with more than one species involved, e.g.

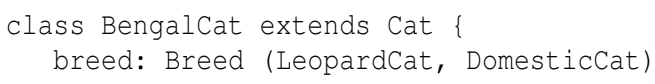

where Breed is defined as

class Breed (list<Species $>$ ) \{\}

It would complicate the notion of ontological metamodeling further to cover this situation.

\section{RELATED WORK}

In this section, we review different ways to look at the term 'model' and in particular different views on M0. We have so far only covered the situations where 'model' is the term used for 'description' of what will be at M0, and thereby implicitly a model of some referent system in some domain. The notion of UML models as blueprints ${ }^{6}$ is very different from this, but in fact supported by section 6.3.1 in the UML 2.5.1 specification $^{7}$ :

For example, for a model of factory processes, the execution scope may encompass the execution of those processes within a single factory, while, for a model of a software program, the execution scope will correspond to a single execution of that program.

For M0, we consider here the following two main approaches: real-world objects and execution instances, see also [Gjøsæter et al., 2016]. For the approaches covered in this section, we judge if they resonate with system development in general and with programmers in particular, because if not, there will be no chance that a given approach will be accepted outside the modeling community.

\footnotetext{
${ }^{6} \mathrm{https}: / /$ martinfowler.com/bliki/UmlAsBlueprint.html

${ }^{7}$ https://www.omg.org/spec/UML/2.5.1/PDF
} 


\section{M0 as Real-world Objects}

In this view, M0 is the area of reality containing "real things" (e.g., real books, passengers and dogs). The other levels belong to the modeling realm being fundamentally different from M0, such that M0 is not part of the metamodel stack [Eriksson et al., 2013]. James Skene states in [Skene, 2007]:

On level M0 are real-world objects. These are described by UML models at level M1. The metamodel of UML is at M2, an instance of the MOF model on level M3.

This view will not resonate at all with Executable UML, where M0 obviously will have instances of classes at M1, not "real things". It also resonates badly with programmers, as they have instances of M1 classes at M0. In this approach, we also find the idea that M1 contains two ontological levels: One for user classes and one for user class objects. See also the discussion in Section 4 about ontological instances. Colin Atkinson and Thomas Kühne state in [Atkinson and Kühne, 2003]:

The M0 level is no longer inhabited by user objects, but rather by the real-world elements that they model. Note that the real Lassie is said to be represented by object Lassie, i.e., instance-of is no longer used to characterize the real Lassie as an instance of Collie. User objects (i.e., model representatives of real-world objects) now occupy the M1 level, along with the types of which they are (ontological) instances. From level M1 on, every level is regarded as a model expressed in the language defined at the level above.

Wikipedia shows the same understanding. The illustration of M0 in Figure 12 is part of the Wikipedia on $\mathrm{UML}^{8}$.

This understanding is said to be based on UML2.3 ${ }^{9}$, but is apparently a misunderstanding, as UML2.3 has runtime instances at M0.

In [Seidewitz, 2003] it is referred to what is said about M0 in MOF1.4 ${ }^{10}$ : M0 is 'What is to be modeled', see figure 13:

This is in contrast to the view put forward in this paper: The real things (phenomena) being modeled are at the level of M0, but outside the stack, and the

\footnotetext{
${ }^{8} \mathrm{https} / / /$ en.wikipedia.org/wiki/Unified_Modeling_Language ${ }^{9} \mathrm{https} / / /$ www.omg.org/spec/UML/2.3/Infrastructure/PDF

${ }^{10}$ Meta Object Facility (MOF) Specification, ver. 1.4, OMG document formal/02-04-03, Object Management Group, Apr. 2002, Section 2.2; www.omg.org/technology/documents/formal/mof.htm
}

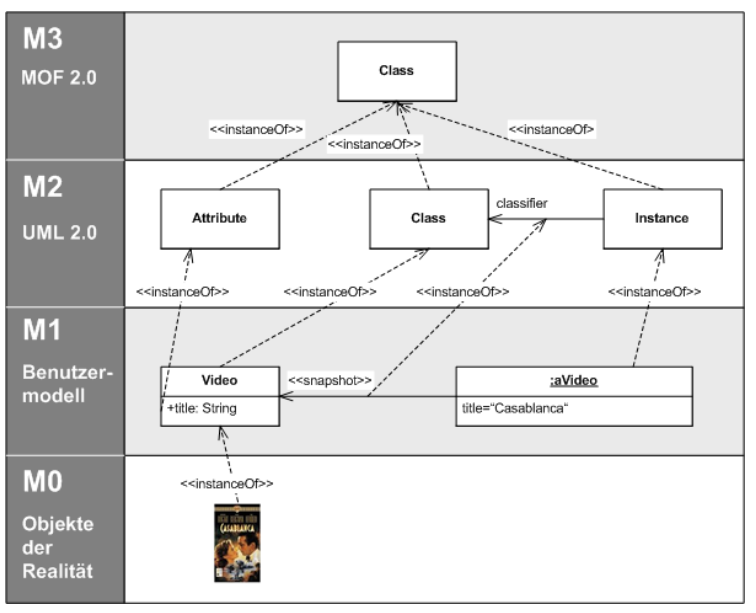

Figure 12: M0 at Wikipedia

M0-What is to be modeled M1-Models (for example, a UML model)

- M2-Metamodels (for example, an abstractsyntax model in the UML specification) M3-The meta-metamodel

Figure 13: M0 for MOF 1.4

objects at M0 model these things. Of course, the objects in executions at M0 are real-world objects in the sense that they physically exist in a computer.

\section{M0 with Execution Instances}

The objects (in general instances) on $\mathrm{M} 0$ are instances of the class descriptions (in general classifiers) at M1. Some use the term data instead of instance; as in a hierarchy where a database schema language is on M2, database schema on M1, and database tables with data on M0. The model (M1) provides a design of the system, and level M0 contains the data objects described at M1. Runtime instances are an extension of this view. Instantiation depends upon the semantics of the language. Colin Atkinson and Thomas Kühne state [Atkinson, 1997]:

Thus, the information at the data level is known as an M0 model, the traditional model level is known as the M1 level, the metamodel level is known as the M2 level, and so on... The M0 level is unique because it can contain no instantiable elements (i.e. templates). It is unique among the levels, therefore, in being populated solely by objects.

The execution instances view is supported by [OMG, 2011, Clark et al., 2004, Bézivin and Gerbé, 2001, 
Favre, 2004]. Jean-Marie Favre states in [Favre, 2003]:

The model level is used to manage the set of all possible real-world situations which are represented at the instance level (M0). For instance "Tom" might be a client that owns two accounts "a4099" and "a2394" with a respective balance of $\$ 800$ and $\$ 2000$. A point at the instance level describes a particular state of a software at a particular point in time. It corresponds to a program state. Program execution indeed corresponds to the evolution of this state.

Jean Bezivin states in [Bézivin and Gerbé, 2001] that M0 corresponds to one given dynamic execution of a program. However, it is unrelated to modeling (it does not contain model elements, but rather real or imaginary situational items and facts). A given execution of a program on level M0 is not itself a model; it is depicted by a model (the source code of the program that describes the infinite number of different executions of the program). Precisely the same situation exists in the four OMG metamodeling layers: M0: the concrete level (any real situation, unique in space and time, represented by a given model from M1).

In [Seidewitz, 2003], the following is said about models.

A models meaning has two aspects. The first is the models relationship to the thing being modeled. This is meaning in the sense of 'This class model means that the Java program must contain these classes.'

This corresponds to the UML-as-blueprint approach mentioned above. The second aspect (according to [Seidewitz, 2003]) is in line with the execution instances approach:

The second aspect is the model's relationship to other models derivable from it. This is meaning in the sense of 'This class model means that instances of these classes are related in this way.' I have called this a theory of the modeling language; this is often called the modeling language's 'semantics' ...

In a presentation ${ }^{11}$ at a workshop on unified modeling and programming ${ }^{12}$ Ed Seidewitz says that 'All

\footnotetext{
${ }^{11} \mathrm{https}: / /$ www.slideshare.net/seidewitz/xumlpresentation-111023-models-programs-and-x-uml, 2011

${ }^{12} \mathrm{https}: / /$ pure.au.dk/portal/en/publications/coomp-2011first-international-workshop-on-combined-objectorientedmodeling-and-programming(77ac2dea-fa3a-49cb-b5b976d6361b447f).html
}

programs are models' 'Executable Models are Programs', and 'Programming in UML is just Programming'. This view is the same as presented here; the only difference is that we term the execution a model. In [Seidewitz, 2016] it is said that Executable UML models and programs are models of executions.

The notion of executable models was coined way before plain UML (1997) and therefore before Executable UML. In 1992, SDL [Union, 2011] was turned into an object-oriented specification language with well-defined execution semantics, exploiting processes with state machines, and in 1994 ROOM [Selic et al., 1994] did the same with processes and Statecharts.

\section{SUMMARY}

This paper has clarified artefacts like models, descriptions and executions, and the relations between these artefacts in systems development.

\section{Executing a system description leads to a system.}

In this paper we have argued that in order to have programmers employ modeling for real, a model has to be the executing system of objects that has a model-of relation to a planned system. Such a model will have a system model prescription, and the execution of this prescription will create the model. In UML, this system model description is called a UML model.

A system can be model-of a referent system. It appears quite obvious in a programming context that some objects (existing during the execution of a program) model the corresponding entities in the application domain. This view applies to modeling as well.

Ontological instantiation relates descriptions. The possibility to describe objects in UML using Instancespecification has created considerable confusion about the levels, about instantiation, and about M0. The idea of models being executions at M0 helps in understanding that there is a major difference between ontological instantiation and linguistic instantiation. Based on this difference, ontological instantiation is only a relation between different description elements: InstanceSpecification and its classifier.

Model and code can describe the same system. A UML model does not model the corresponding Java code, but both are descriptions of systems that might model some referent system. They can 
describe the same system on different levels of abstraction.

These clarifications contribute to the alignment of programming and modeling. This paper has just touched upon semantics and meaning; an in-depth coverage of semantics and meaning, which are closely related to M0, is planned for a future article.

\section{REFERENCES}

Aldrich, J., Chambers, C., and Notkin, D. (2002). Archjava: Connecting software architecture to implementation. In Proceedings of the 24th International Conference on Software Engineering, ICSE '02, pages 187-197, New York, NY, USA. ACM.

Aßmann, U., Zschaler, S., and Wagner, G. (2006). Ontologies, meta-models, and the model-driven paradigm. In Calero C., Ruiz F., Piattini M. (eds): Ontologies for Software Engineering and Software Technology. Springer Berlin Heidelberg.

Atkinson, C. (1997). Meta-modeling for distributed object environments. In In Enterprise Distributed Object Computing, pages 90-101. Published by IEEE Computer Society.

Atkinson, C. and Kühne, T. (2003). Model-driven development: A metamodeling foundation. Software, IEEE.

Bézivin, J. and Gerbé, O. (2001). Towards a Precise Definition of the OMG/MDA Framework. Proceedings of ASE'01, Automated Software Engineering.

Bossel, H. (2013). Modeling and Simulation. Vieweg+Teubner Verlag.

Butting, A., Jansen, N., Rumpe, B., and Wortmann, A. (2018). Translating grammars to accurate metamodels. In Proceedings of the International Conference on Software Language Engineering (SLE'18).

Clark, T., Evans, A., Sammut, P., and Williams, J. (2004). Applied Metamodelling. A Foundation for Language Driven Development. Xactium. Available at http: / / www. xactium.com.

Daun, M., Brings, J., Krajinski, L., and Weyer, T. (2019). On the benefits of using dedicated models in validation processes for behavioral specifications. In Proceedings of IEEE/ACM Internatioal Conference and System Processes (ICSSP).

Eriksson, O., Henderson-Sellers, B., and Ågerfalk, P. J. (2013). Ontological and linguistic metamodelling revisited: A language use approach. Information and Software Technology.

Exner, K., Lindowa, K., Buchholz, C., and Stark, R. (2014). Validation of product-service systems a prototyping approach. In Proceedings of 6th CIRP Conference on Industrial Product-Service Systems.

Favre, J.-M. (2003). Meta-model and model co-evolution within the 3D software space. In Proceedings of ELISA 2003.

Favre, J.-M. (2004). Foundations of meta-pyramids: Languages vs. metamodels - episode ii: Story of thotus the baboon. In Language Engineering for Model-Driven Software Development.

Fischer, J., Møller-Pedersen, B., and Prinz, A. (2016). Modelling of systems for real. In Proceedings of the 4th International Conference on Model-Driven Engineering and Software Development, pages 427-434.

Fowler, M. (2005). Language workbenches: The killer-app for domain specific languages? http://www.martinfowler.com/articles/language Workbench.html.

Gjøsæter, T., Prinz, A., and Nytun, J. P. (2016). MOF-VM: Instantiation revisited. In Proceedings of the 4th International Conference on Model-Driven Engineering and Software Development, pages 137-144.

Holbæk-Hanssen, E., Håndlykken, P., and Nygaard, K. (1973). System description and the delta language. Technical report, Oslo: Norwegian Computing Center.

Kawata, S. (2015). Computer Assisted Problem Solving Environment (PSE), pages 1251-1260. IGI Global.

Kühne, T. (2006). Matters of (meta-) modeling. Software and Systems Modeling (SoSyM), 5(4):369-385.

Madsen, O. L. and Møller-Pedersen, B. (2018). This is not a model : On development of a common terminology for modeling and programming. In Proceedings of the 8th International Symposium, ISoLA 2018: Leveraging Applications of Formal Methods, Verification and Validation - Modeling, Lecture Notes in Computer Science 2018 ;Volume 11244 LNCS, pages 206-224.

OMG (2011). Unified Modeling Language: Infrastructure version 2.4.1 (OMG Document formal/2011-08-05). OMG Document. Published by Object Management Group, http://www.omg.org.

OMG (2014). Ontology Definition Metamodel Version 1.1. Technical report, Object Management Group.

OMG (2016). Meta Object Facility (MOF). Technical report, Object Management Group.

OMG (2018). Semantics of a Foundational Subset for Executable UML Models (fUML). Technical report, Object Management Group.

Prinz, A., Møller-Pedersen, B., and Fischer, J. (2016). Object-oriented operational semantics. In Proceedings of SAM 2016, LNCS 9959, Berlin, Heidelberg. Springer-Verlag.

Seidewitz, E. (2003). What models mean. IEEE Software.

Seidewitz, E. (2016). On a unified view of modeling and programming, position paper. In Proceedings of ISoLA 2016.

Selic, B., Gullekson, G., and Ward, P. T. (1994). Real-time Object-oriented Modeling. John Wiley \& Sons, Inc., New York, NY, USA.

Skene, J. (2007). Language Support for Service-Level Agreements for Application-Service Provision. PhD thesis, University of London. Accessed January, 2015: http://eprints.ucl.ac.uk/5607/1/5607.pdf.

Union, I. T. (2011). Z.100 series, specification and description language sdl. Technical report, International Telecommunication Union.

W3C (2012). OWL 2 Web Ontology Language. Technical report, W3C. 